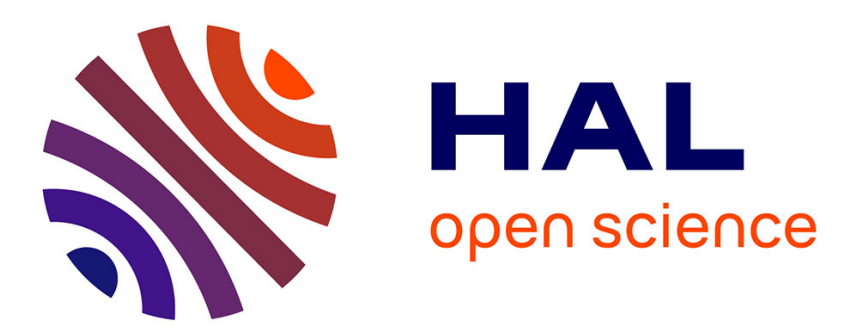

\title{
Comparison between classes of state-quadratic Lyapunov functions for discrete-time linear polytopic and switched systems
}

Paolo Mason, Mario Sigalotti, Jamal Daafouz

\section{- To cite this version:}

Paolo Mason, Mario Sigalotti, Jamal Daafouz. Comparison between classes of state-quadratic Lyapunov functions for discrete-time linear polytopic and switched systems. Systems and Control Letters, 2012, 61 (11), pp.1062-1068. 10.1016/j.sysconle.2012.07.010 . inria-00629245v2

\section{HAL Id: inria-00629245 \\ https://hal.inria.fr/inria-00629245v2}

Submitted on 30 Jul 2012

HAL is a multi-disciplinary open access archive for the deposit and dissemination of scientific research documents, whether they are published or not. The documents may come from teaching and research institutions in France or abroad, or from public or private research centers.
L'archive ouverte pluridisciplinaire HAL, est destinée au dépôt et à la diffusion de documents scientifiques de niveau recherche, publiés ou non, émanant des établissements d'enseignement et de recherche français ou étrangers, des laboratoires publics ou privés. 


\title{
Comparison between classes of state-quadratic Lyapunov functions for discrete-time linear polytopic and switched systems *
}

\author{
Paolo Mason ${ }^{\dagger}$, Mario Sigalotti ${ }^{\ddagger}$, Jamal Daafouz ${ }^{\S}$
}

July 30, 2012

\begin{abstract}
The paper deals with the stability properties of linear discrete-time switched systems with polytopic sets of modes. The most classical way of studying the uniform asymptotic stability of such a system is to check for the existence of a quadratic Lyapunov function. It is known from the literature that letting the Lyapunov function depend on the time-varying switching parameter improves the chance that a quadratic Lyapunov function exists. Our objective is to compare different notions of quadratic stability. The contribution of this paper is twofold. In the first part we consider switching systems satisfying a certain non-degeneracy assumption and we prove that, for such systems, no gain in the stability analysis is obtained if we allow the Lyapunov function to depend explicitly also on time. In the second part we consider the case where the non-degeneracy assumption is violated. We prove that in this case allowing the Lyapunov function to depend on time is less conservative. We also show that new LMI conditions can be used in order to characterize the existence of a time-dependent quadratic Lyapunov function. Moreover in the paper we discuss the case where the variation of the switching parameter is bounded by a prescribed constant between two subsequent times.
\end{abstract}

Keywords: discrete-time; linear switched system; mode-dependent Lyapunov function; quadratic Lyapunov function; linear matrix inequality.

\section{Introduction}

This paper is devoted to linear discrete-time systems

$$
x(k+1)=A_{\xi(k)} x(k),
$$

*This work was supported by the French ANR grant ArHyCo, Programme ARPEGE, contract number ANR-2008 SEGI 004 01-30011459.

${ }^{\dagger}$ P. Mason is with CNRS-LSS-Supélec, 3 rue Joliot-Curie, 91192 Gif-sur-Yvette, France. E-mail: paolo.mason@lss.supelec.fr.

${ }^{\ddagger}$ M. Sigalotti is with INRIA Saclay-Île-de-France, Team GECO, and CMAP, UMR 7641, École Polytechnique, Route de Saclay, 91128 Palaiseau Cedex, France. E-Mail: mario.sigalotti@inria.fr.

$\S$ J. Daafouz is with CRAN, 2 avenue de la forêt de Haye, 54516 Vandœuvre-lès-Nancy cedex, France. E-mail: Jamal.Daafouz@ensem.inpl-nancy.fr. 
where $\xi(k) \in \Xi \subset \mathbf{R}^{m}$ and $x(k) \in \mathbf{R}^{d}$ for every $k \in \mathbf{N}$. We will refer to $k \mapsto \xi(k)$ as to a switching function $\left(\xi(k)\right.$ being the switching parameter). We denote by $A_{\Xi}$ the set of admissible modes, i.e., $A_{\Xi}=\left\{A_{\xi} \mid \xi \in \Xi\right\}$. Most of the paper will deal either with the case where $A_{\Xi}$ is finite or with the case where $A_{\Xi}$ is a convex polytope, i.e., the convex hull of finitely many matrices. Dynamical systems described by (1) with $A_{\Xi}$ a convex polytope are also called polytopic discrete-time systems in the literature [6, 14].

Historically, the stability of this class of dynamical systems has been analyzed using the concept of quadratic stability, which we shall call static quadratic stability to avoid confusion with what follows. This notion was inspired by [4] where Lyapunov functions quadratic in the state and independent of the switching parameter were used for the first time. The main advantage in using such particular Lyapunov functions is the fact that necessary and sufficient conditions for quadratic stability can be formulated in terms of algebraic Riccati equations or linear matrix inequalities (LMIs) [8]. The available solvers make the solutions proposed in this context numerically tractable. Looking for more general Lyapunov functions has received special attention during the last decades in order to derive checkable stability conditions that are more general than those based on static quadratic stability. LMI stability conditions using Lyapunov functions quadratic in the state but with a linear dependence with respect to the switching parameter have been developed in [11]. These conditions are proved to be necessary and sufficient for the existence of this kind of Lyapunov functions and can also be used for design problems (control, state reconstruction, etc). At the expense of more computational effort, less conservative conditions have been proposed in [17] using quadratic Lyapunov functions depending on several past values of the switching parameters. Advances in the classification of sufficient LMI conditions for stability have been proposed in [1]. In recent years, stability analysis has also been carried out in the framework of the so-called joint spectral radius, a measure of the maximal asymptotic growth rate [7, 16]. Despite its natural interpretation and the fact that it leads to a necessary and sufficient stability condition, the joint spectral radius is difficult to compute. A procedure for approximating the joint spectral radius with arbitrary high accuracy is provided for the case of finite sets of matrices; however, of course, higher accuracy comes at larger computational cost.

A question of interest is the following: can one expect an improvement of the results in [11] by considering other quadratic Lyapunov functions (not necessarily linear with respect to the switching parameter and not necessarily time-independent)? In the case of linear time-varying (LTV) systems $(k \mapsto \xi(k)$ fixed) it is known that stability is equivalent to the existence of a time-varying quadratic Lyapunov function (see [24]). For LTV's, therefore, time-varying quadratic Lyapunov functions are not equivalent to time-invariant ones as a tool to check stability. Answering whether this is still the case for switched linear discrete-time systems does not seem to be immediate. To this end, we focus in this paper on three criteria of stability. The first one is called Parameter Dependent quadratic stability (PD-quadratic stability). It refers to checking stability by mean of Lyapunov function quadratic in the state and dependent on the switching parameter but without any specified structure. The second one is called Parameter and Time Dependent quadratic stability (PTD-quadratic stability). It refers to Lyapunov functions that are quadratic in the state and depend explicitly on both the time and the parameters. The last one, and a priori the less costlier to check, is the so-called poly-quadratic stability used in [11] and which refers to Lyapunov functions quadratic in the state and linear in the switching parameter.

The contribution of this paper is twofold. First, we prove that all these criteria are equiva- 
lent in the non-degenerate case, namely when there exists $\bar{\xi} \in \Xi$ such that $A_{\bar{\xi}}$ is invertible (see Proposition 4 and Theorem 6). Such an assumption is not restrictive when (1) is obtained by discretization of a continuous-time system. Second, in the degenerate case, we introduce the notion of eventual accessible sets and we show that it leads to a relaxation of the LMI conditions to check stability of switched linear systems that are not PD-quadratically stable (see Theorem 14). We also discuss the case where parameter variation is bounded.

It is known that available necessary and sufficient stability conditions for switched systems (such as the joint spectral radius [16] or those based on Lyapunov functions for difference inclusions $[3,20]$ ) are difficult to check numerically. A popular approach has been to try to approximate these conditions by a class of conditions that we can efficiently solve using convex optimization and in particular semidefinite programming. Semidefinite programs (SDPs) can be solved with arbitrary accuracy in polynomial time and lead to efficient computational methods. Our above-mentioned contributions imply the following: (i) in the non-degenerate case it is redundant (and numerically costlier) to consider time-dependent Lyapunov functions or Lyapunov functions which depend nonlinearly on the switching parameter; (ii) in the degenerate case time-dependent quadratic Lyapunov functions give a less conservative test for stability (see Example 17), which can still be formulated in terms of LMIs.

The paper is organized as follows. In Section 2 we introduce the main definitions and we discuss the equivalence of asymptotic stability under convexification of the set of state-space matrices. In Section 3.1, we prove the equivalence between the stability criteria introduced above and we discuss the case where parameter variation is bounded. We also illustrate in the discrete time setting the well-known fact that uniformly asymptotically stable switched systems which do not admit quadratic Lyapunov functions exist showing that a system can be uniformly asymptotically stable without being poly-quadratically stable (or PD-quadratically stable, nor PTD-quadratically stable). The notion of eventual accessible sets and the relaxed LMIs conditions are introduced in Section 4. This allows to compare the previous notions of quadratic stability in the general case. We end the paper by a conclusion.

\section{Preliminaries}

Fix $d \in \mathbf{N}$. Let $\left\{e_{1}, \ldots, e_{d}\right\}$ be the canonical basis of $\mathbf{R}^{d}$ and denote by $M_{d}(\mathbf{R})$ the set of all real $d \times d$ matrices. Recall that a partial order on $M_{d}(\mathbf{R})$ is defined as follows: Given $A, B \in M_{d}(\mathbf{R})$, we write $A \leq B$ if $A-B$ is negative semidefinite. A function with values in $M_{d}(\mathbf{R})$ is said to be convex if it is so with respect to such order. We say that $A>0$ on a subset $\Sigma$ of $\mathbf{R}^{d}$ if $x^{T} A x>0$ for every $x \in \Sigma \backslash\{0\}$. The Euclidian norm in $\mathbf{R}^{d}$ and that induced in $M_{d}(\mathbf{R})$ are both denoted by $\|\cdot\|$. A function $w: \varepsilon \mapsto w(\varepsilon) \in M_{d}(\mathbf{R})$ defined for all $\varepsilon>0$ is said to be of order $k(k \in \mathbf{N})$ if $\limsup _{\varepsilon \rightarrow 0}\|w(\varepsilon)\| \varepsilon^{-k}$ is finite. In this case we write $w(\varepsilon)=\mathcal{O}\left(\varepsilon^{k}\right)$.

Let $m \in \mathbf{N}, \Xi$ be a subset of $\mathbf{R}^{m}$ and $A: \xi \mapsto A_{\xi}$ be a map from $\Xi$ to $M_{d}(\mathbf{R})$. As stated in the introduction, we consider the dynamical system (1) with $A_{\Xi}$ the set of admissible modes, i.e., $A_{\Xi}=\left\{A_{\xi} \mid \xi \in \Xi\right\}$. Without loss of generality, in the case where $A_{\Xi}$ is finite, we assume that $m=1$ and $\Xi=\{1, \ldots, M\}$ for some $M \in \mathbf{N}$ so that $A_{\Xi}=\left\{A_{1}, \ldots, A_{M}\right\}$ and we say that (1) is finite. In the case where $A_{\Xi}$ is a convex polytope, we denote by $\left\{A_{1}, \ldots, A_{M}\right\}$ the set of its vertices and we assume that $m=M, \Xi=\operatorname{conv}\left\{e_{1}, \ldots, e_{M}\right\}$, and that $A$ is the linear map satisfying $A_{i}=A_{e_{i}}$. In this case we say that system (1) is polytopic. 
The notion of uniform asymptotic stability of a discrete-time switched system is recalled in the following definition.

Definition 1 We say that (1) is uniformly asymptotically stable $(U A S)$ if for every $x(0) \in \mathbf{R}^{d}$ the solution to (1) converges to zero uniformly with respect to $\{\xi(k)\}_{k \in \mathbf{N}} \subset \Xi$ (i.e., for every $\varepsilon>0$ there exists $K \in N$ such that for every $\{\xi(k)\}_{k \in \mathbf{N}} \subset \Xi$ we have $\|x(k)\|<\varepsilon$ for $k \geq K)$ and if, moreover, for every $R>0$ there exists $r>0$ such that $\|x(k)\|<R$ for every $\{\xi(k)\}_{k \in \mathbf{N}} \subset \Xi$ and every $k \in \mathbf{N}$, provided that $\|x(0)\|<r$.

Because of the linear nature of the dynamics of (1), it is well known that (1) is uniformly exponentially stable if and only if it is UAS (indeed, if ad only if the origin is attractive, see for instance $[10, \S 5.2])$.

\subsection{Lyapunov functions}

A classical sufficient condition for the UAS of (1) is the existence of a (parameter-dependent) quadratic Lyapunov function.

Definition 2 We say that (1) is parameter-dependent quadratically stable (PD-quadratically stable) if there exist three positive constants $\alpha_{0}, \alpha_{1}, \alpha_{2}$ and a Lyapunov function

$$
V(x, \xi)=x^{T} P_{\xi} x
$$

with $\Xi \ni \xi \mapsto P_{\xi} \in \mathcal{M}^{d \times d}$ such that

$$
\alpha_{1}\|x\|^{2} \leq V(x, \xi) \leq \alpha_{2}\|x\|^{2}, \quad x \in \mathbf{R}^{d}, \quad \xi \in \Xi,
$$

and

$$
V\left(A_{\xi} x, \eta\right)-V(x, \xi) \leq-\alpha_{0}\|x\|^{2}, \quad x \in \mathbf{R}^{d}, \quad \xi, \eta \in \Xi .
$$

We recall below the notion of poly-quadratic stability, introduced in [11], which corresponds to the special case where, in the definition above, system (1) is polytopic and $V$ is linear with respect to $\xi$.

Definition 3 Let (1) be polytopic. We say that (1) is poly-quadratically stable if there exists a function $V$ satisfying (2), (3) and (4) that is linear with respect to $\xi$, i.e., $P_{\xi}=\sum_{i=1}^{M} \xi_{i} P_{e_{i}}$.

For polytopic systems it turns out that, on the one hand, UAS is equivalent to the same property for the system having as modes the vertices of $A_{\Xi}$ and, on the other hand, PDquadratic stability and poly-quadratic stability are equivalent. These facts are detailed in the following proposition, which is essentially a collection of known results.

Proposition 4 Let (1) be polytopic. Then (1) is UAS if and only if the finite system with modes $\left\{A_{1}, \ldots, A_{M}\right\}$ is UAS. Moreover, (1) is poly-quadratically stable if and only if there exist a scalar $\alpha_{0}>0$ and $M$ symmetric matrices $P_{1}, \ldots, P_{M}$ such that

$$
\begin{gathered}
P_{i}>0 \quad \forall i \in\{1, \ldots, M\} \\
A_{i}^{T} P_{j} A_{i}-P_{i} \leq-\alpha_{0} \mathrm{Id}, \quad \forall i, j \in\{1, \ldots, M\}
\end{gathered}
$$

In particular, (1) is poly-quadratically stable if and only if it is PD-quadratically stable. 
Proof. The first part of the statement follows from classical results characterizing stability of discrete-time switched systems in terms of the joint spectral radius. It is well known that for a bounded set of matrices $A_{\Xi}$ the uniform asymptotic stability of (1) is equivalent to the property that the joint spectral radius

$$
\rho\left(A_{\Xi}\right)=\limsup _{h \rightarrow \infty} \max _{\xi^{1}, \ldots, \xi^{h} \in \Xi}\left\|A_{\xi^{1}} \cdots A_{\xi^{h}}\right\|^{\frac{1}{h}}
$$

is strictly smaller than one. The claimed equivalence then follows from the equality

$$
\rho\left(\operatorname{conv}\left\{A_{1}, \ldots, A_{M}\right\}\right)=\rho\left(\left\{A_{1} \ldots A_{M}\right\}\right),
$$

which has been observed in [23] (see also [16, 22] and, for related discussions [5, 18]).

The second part of the statement, which expresses poly-quadratic stability in terms of finitely many LMIs, trivially follows from [11]. (See also [12, Theorem 2].)

Finally, concerning the last part of the statement, PD-quadratic stability implies by definition conditions (5)-(6) and therefore poly-quadratic stability.

Motivated by the characterization of asymptotic stability of LTV's in terms of existence of time-varying quadratic Lyapunov functions (see [24]), we introduce the following (a priori weaker) notion.

Definition 5 We say that (1) is parameter-and time-dependent quadratically stable (PTDquadratically stable) if there exist three positive constants $\alpha_{0}, \alpha_{1}, \alpha_{2}$ and a Lyapunov function

$$
V(k, x, \xi)=x^{T} P_{k, \xi} x
$$

such that

$$
\alpha_{1}\|x\|^{2} \leq V(k, x, \xi) \leq \alpha_{2}\|x\|^{2}, \quad x \in \mathbf{R}^{d}, \quad \xi \in \Xi,
$$

and for every $x(0) \in \mathbf{R}^{d}$, every $\{\xi(k)\}_{k \in \mathbf{N}} \subset \Xi$, and every $k \in \mathbf{N}$, we have

$$
V(k+1, x(k+1), \xi(k+1))-V(k, x(k), \xi(k)) \leq-\alpha_{0}\|x(k)\|^{2} .
$$

\section{The nondegenerate case}

In this section we investigate quadratic stability under the hypothesis that a mode of system (1) is nondegenerate. Notice that such hypothesis is very natural since it is always satisfied when (1) is obtained by discretization of a continuous-time system. We first compare the notions introduced in the previous section and we then adapt the result to systems satisfying particular constraints on the switching laws.

\subsection{Equivalence between different notions of quadratic stability}

The following theorem states the equivalence between the three notions of quadratic stability introduced in the previous section, under the hypothesis that a mode of system (1) is nondegenerate. The case where the hypothesis does not necessarily hold is considered in Section 4. Motivated by the first part of Proposition 4, we state the result only for finite systems. A straightforward adaptation to the polytopic case is given in Remark 7. 
Theorem 6 Let (1) be finite. Assume that there exists $\bar{\xi} \in \Xi$ such that $A_{\bar{\xi}}$ is invertible. Then (1) is PTD-quadratically stable if and only if it is PD-quadratically stable.

Proof. It is clear by the definitions given in Section 2 that if (1) is PD-quadratically stable then it is PTD-quadratically stable. (Notice that we do not need, for this part of the argument, to assume the existence of $\bar{\xi} \in \Xi$ such that $\operatorname{det}\left(A_{\bar{\xi}}\right) \neq 0$.)

Assume then that (1) is PTD-quadratically stable and fix $(k, \xi, x) \mapsto V(k, \xi, x)$ and $(k, \xi) \mapsto P_{k, \xi}$ as in Definition 5. We are left to prove that (1) is PD-quadratically stable.

Given $k \in \mathbf{N}$, take $\xi(j)=\bar{\xi}$ for $j<k$ so that for every $\bar{x} \in \mathbf{R}^{d}$ we can choose $x(0)$ in such a way that the solution of (1) satisfies $x(k)=\bar{x}$. Considering any choice of $\xi(k), \xi(k+1)$ in $\{1, \ldots, M\}$, we deduce from (9) and the arbitrariness of $\bar{x}$ that

$$
A_{i}^{T} P_{k+1, j} A_{i}-P_{k, i} \leq-\alpha_{0} \mathrm{Id}, \quad i, j \in\{1, \ldots, M\} .
$$

Define $\Omega(k)=\left(P_{k, 1}, \ldots, P_{k, M}\right)$ for every $k \in \mathbf{N}$. Notice that $\{\Omega(k)\}_{k \in \mathbf{N}}$ is a bounded sequence in $\left(M_{d}(\mathbf{R})\right)^{M}$, due to (8). We can thus extract a converging subsequence $\left\{\Omega\left(k_{l}\right)\right\}_{l \in \mathbf{N}}$.

For every $l \in \mathbf{N}$, let us consider a time-independent $M$-uple of symmetric positive definite matrices of the form

$$
P_{j}^{l, *}=\sum_{k=k_{l}}^{k_{l+1}-1} P_{k, j}, \quad j \in\{1, \ldots, M\} .
$$

Taking $l$ large enough, we can assume

$$
-\frac{\alpha_{0}}{2} \mathrm{Id} \leq A_{i}^{T}\left(P_{k_{l+1}, j}-P_{k_{l}, j}\right) A_{i} \leq \frac{\alpha_{0}}{2} \mathrm{Id}, \quad i, j \in\{1, \ldots, M\} .
$$

Then, for every $i, j \in\{1, \ldots, M\}$ and every $l$ large enough,

$$
\begin{aligned}
A_{i}^{T} P_{j}^{l, *} A_{i}-P_{i}^{l, *} & =A_{i}^{T} P_{k_{l}, j} A_{i}-P_{k_{l+1}-1, i}+\sum_{k=k_{l}}^{k_{l+1}-2}\left(A_{i}^{T} P_{k+1, j} A_{i}-P_{k, i}\right) \\
& \leq A_{i}^{T}\left(P_{k_{l}, j}-P_{k_{l+1}, j}\right) A_{i}+\sum_{k=k_{l}}^{k_{l+1}-1}\left(A_{i}^{T} P_{k+1, j} A_{i}-P_{k, i}\right) \\
& \leq-\left(k_{l+1}-k_{l}-\frac{1}{2}\right) \alpha_{0} \operatorname{Id} \leq-\frac{\alpha_{0}}{2} \mathrm{Id} .
\end{aligned}
$$

Setting $\hat{\alpha}=\alpha_{0} / 2$ and $P_{i}^{*}=P_{i}^{l, *}$ for $i=1, \ldots, M$ and $l$ large (independent of $i$ and $j$ ), we have

$$
A_{i}^{T} P_{j}^{*} A_{i}-P_{i}^{*} \leq-\hat{\alpha} \mathrm{Id}, \quad i, j \in\{1, \ldots, M\},
$$

which concludes the proof of Theorem 6 .

Remark 7 Theorem 6 can be extended to the case where (1) is polytopic. Indeed, assume that there exists $\bar{\xi} \in \Xi=\operatorname{conv}\left(e_{1}, \ldots, e_{M}\right)$ such that $\operatorname{det} A_{\bar{\xi}} \neq 0$ and that (1) is PTD-quadratically stable. Then the finite system having $A_{e_{1}}, \ldots, A_{e_{M}}, A_{\bar{\xi}}$ as modes is PTD-quadratically stable and, by Theorem 6, PD-quadratically stable. It follows by Proposition 4 that (1) is PDquadratically stable. 


\section{$3.2 \quad \delta$-stability}

We consider in this section the problem of detecting through quadratic Lyapunov functions the stability of polytopic systems whose switching functions have some common bound on the speed of variation. More precisely, given $\delta>0$, we say that $\xi: \mathbf{N} \rightarrow \Xi$ is a $\delta$-switching function if $\|\xi(k+1)-\xi(k)\|<\delta$ for every $k \in \mathbf{N}$. This constraint has a practical justification. Indeed, the class of dynamical systems we consider here is also studied in the context of the so-called linear parameter varying (LPV) systems where a scheduling parameter is assumed to vary arbitrarily within a polytopic set. In practice, however, there are often limitations on the rate of parameter variation. Two of many examples for limited-variation parameters in LPV systems are the amount of fuel in an airplane for flight control systems or the engine speed in engine control systems. Taking these limitations into account leads to less conservative results. This fact has been recognized in the literature and is incorporated in other control methods for LPV systems (see $[2,9,15]$ and references therein).

We say that (1) is $\delta$-UAS if it is uniformly asymptotically stable with respect to the class of $\delta$-switching functions. Analogously, the notion of PTD-quadratic stability admits a straightforward counterpart for $\delta$-switching functions: we speak of $\delta$-PTD-quadratic stability. As for PD-quadratic stability, we can define the corresponding notion of $\delta$-PD-quadratic stability by replacing (4) by

$$
V\left(A_{\xi} x, \eta\right)-V(x, \xi) \leq-\alpha_{0}\|x\|^{2}, \quad x \in \mathbf{R}^{d}, \quad \xi, \eta \in \Xi, \quad\|\xi-\eta\|<\delta .
$$

Finally, in order to extend the notion of poly-quadratic stability to the case of a polytopic system (1), we replace the assumption that the Lyapunov function is linear on $\Xi$ by the requirement that it is just piecewise affine, in the following sense. We say that $(1)$ is $(\delta, \rho)$ poly-quadratically stable if it is $\delta$-PD-quadratically stable with a Lyapunov function which is continuous on $\Xi$ and affine on every simplex of a tessellation of $\Xi$ whose simplices have all diameter smaller than $\rho$. We recall that a simplex of dimension $n$ is a polytope with $n+1$ vertices $w_{1}, \ldots, w_{n+1}$ such that the vectors $w_{1}-w_{n+1}, w_{2}-w_{n+1}, \ldots, w_{n}-w_{n+1}$ are linearly independent, while a tessellation by simplices of $\Xi$ is a finite covering of $\Xi$ by simplices of dimension $M-1$ (the dimension of $\Xi$ ) whose (relative) interiors are pairwise disjoint. Finally, the diameter of a subset $S$ of $\mathbf{R}^{M}$ is defined as $\sup _{x, y \in S}\|x-y\|$.

The following holds.

Theorem 8 Let (1) be polytopic and assume that there exists $\bar{\xi} \in \Xi$ such that $A_{\bar{\xi}}$ is invertible. If $(1)$ is $(\delta, \rho)$-poly-quadratically stable then it is $\delta$-PD-quadratically stable and $\delta$-PTDquadratically stable. Moreover, if (1) is $\delta$-PTD-quadratically stable then, for every $\delta^{\prime} \in(0, \delta)$, there exists $\rho>0$ such that $(1)$ is $\left(\delta^{\prime}, \rho\right)$-poly-quadratically stable.

Proof. The first part of the statement being trivial, assume that (1) is $\delta$-PTD-quadratically stable and fix $\alpha_{0}, \alpha_{1}, \alpha_{2}>0,(k, \xi) \mapsto P_{k, \xi}$ and $V(k, x, \xi)=x^{T} P_{k, \xi} x$ such that $\alpha_{1} \mathrm{Id} \leq P_{k, \xi} \leq$ $\alpha_{2}$ Id for every $\xi \in \Xi$ and

$$
V(k+1, x(k+1), \xi(k+1))-V(k, x(k), \xi(k)) \leq-\alpha_{0}\|x(k)\|^{2}, \quad k \in \mathbf{N},
$$

for every solution $x(\cdot)$ of $(1)$ corresponding to a $\delta$-switching function $\xi(\cdot)$.

Fix $\delta^{\prime}$ belonging to $(0, \delta)$. Choose $\rho>0$ such that

$$
\delta^{\prime}+2 \rho<\delta
$$


Fix a tessellation of $\Xi$ such that the diameter of each of its simplices is smaller than $\rho$. Denote by $\mathcal{T}$ the set of simplices of the tessellation and by $\Lambda$ the set of its vertices.

Since the function $\xi \mapsto \operatorname{det}\left(A_{\xi}\right)$ is analytic and, by hypothesis, it does not vanish identically, we deduce that for almost every $\xi \in \Xi$ the matrix $A_{\xi}$ is invertible. Hence, for every $\hat{x} \in \mathbf{R}^{d}$, every $\hat{\xi} \in \Xi$ and every $k \in \mathbf{N}$ there exist $x(0)$ and a $\delta$-switching function $\xi: \mathbf{N} \rightarrow \Xi$ such that the corresponding trajectory $x(\cdot)$ of $(1)$ satisfies $x(k)=\hat{x}, \xi(k)=\hat{\xi}$.

Following the same argument as in the proof of Theorem 6 we can show that there exist $\hat{\alpha}>0$ and a family of positive definite matrices $P_{e}^{*}, e \in \Lambda$, such that

$$
A_{\xi}^{T} P_{\eta}^{*} A_{\xi}-P_{\xi}^{*} \leq-\hat{\alpha} \mathrm{Id} \quad \text { on } \mathbf{R}^{d}
$$

for every $\xi, \eta \in \Lambda$ such that $\|\xi-\eta\|<\delta$.

Extend $P^{*}$, seen as a matrix-valued function defined on $\Lambda$, to the piecewise affine function $\Pi$ on $\Xi$ defined by

$$
\Pi_{\xi}=\sum_{i=1}^{M} \lambda_{i} P_{e_{i}^{\xi}}^{*},
$$

where $e_{1}^{\xi}, \ldots, e_{M}^{\xi} \in \Lambda$ are the vertices of a simplex in $\mathcal{T}$ and $\sum_{i=1}^{M} \lambda_{i} e_{i}^{\xi}=\xi$ with $\sum_{i=1}^{M} \lambda_{i}=1$, $\lambda_{i} \geq 0$ for $i=1, \ldots, M$.

Consider $\xi$ and $\eta$ in $\Xi$ such that $\|\xi-\eta\|<\delta^{\prime}$. Take $e_{1}^{\xi}, \ldots, e_{M}^{\xi}, e_{1}^{\eta}, \ldots, e_{M}^{\eta} \in \Lambda$ as above, with $\sum_{i=1}^{M} \lambda_{i} e_{i}^{\xi}=\xi$ and $\sum_{i=1}^{M} \mu_{i} e_{i}^{\eta}=\eta$. Recall that $\rho$ has been chosen in such a way that (13) holds true. Hence $\left\|e_{i}^{\xi}-e_{j}^{\eta}\right\|<\delta$ for every $i, j=1, \ldots, M$.

Let $j$ belong to $\{1, \ldots, M\}$. Notice that the map $M_{d}(\mathbf{R}) \ni C \mapsto C^{T} P_{e_{j}^{\eta}}^{*} C$ is convex. Hence,

$$
\begin{aligned}
A_{\xi}^{T} P_{e_{j}^{\eta}}^{*} A_{\xi}-\Pi_{\xi}=\left(\sum_{i=1}^{M} \lambda_{i} A_{e_{i}^{\xi}}^{T}\right) P_{e_{j}^{\eta}}^{*} \sum_{i=1}^{M} \lambda_{i} A_{e_{i}^{\xi}}-\sum_{i=1}^{M} \lambda_{i} P_{e_{i}^{\xi}}^{*} & \leq \\
\sum_{i=1}^{M} \lambda_{i}\left(A_{e_{i}^{\xi}}^{T} P_{e_{j}^{\eta}}^{*} A_{e_{i}^{\xi}}-P_{e_{i}^{\xi}}^{*}\right) & \leq-\hat{\alpha} \mathrm{Id}
\end{aligned}
$$

where the last inequality follows from (14). Since the above inequality holds for every $j$, we conclude that

$$
A_{\xi}^{T}\left(\sum_{j=1}^{M} \mu_{j} P_{e_{j}^{\eta}}^{*}\right) A_{\xi}-\Pi_{\xi}=A_{\xi}^{T} \Pi_{\eta} A_{\xi}-\Pi_{\xi} \leq-\hat{\alpha} \mathrm{Id},
$$

as required.

Remark 9 The proof of the theorem (see, in particular, (13)) shows the following tradeoff in the choice of $\delta^{\prime}$ and $\rho$, for a given $\delta$-PTD-quadratically stable system: As $\delta^{\prime}$ gets close to $\delta$, $\rho$ gets small in general; conversely, decreasing $\delta^{\prime}$, we can increase $\rho$, reducing the number of LMIs to be tested.

\subsection{Asymptotic vs quadratic stability}

We go back here to the general case, without bounds on the speed of variation of the switching parameter $\xi$. As already mentioned, the equivalent conditions appearing in the statement of 
Theorem 6 are sufficient for the uniform asymptotic stability of (1). However, they are not necessary. A numerical evidence for this fact was already given in [17], where the authors generalize the notion of PD-quadratic stability by considering quadratic Lyapunov functions depending on several past values of the switching parameters, that is,

$$
V(x(k), \xi(k), \xi(k-1), \ldots, \xi(k-m))=x(k)^{T} P_{\xi(k), \xi(k-1), \ldots, \xi(k-m)} x(k) .
$$

It is proved in [17, Theorem 9] that UAS is equivalent to the existence of $m \in \mathbf{N}$ and $\left(\xi_{0}, \ldots, \xi_{m}\right) \mapsto P_{\xi_{0}, \ldots, \xi_{m}}>0$ satisfying the LMIs

$$
A_{\xi_{0}}^{T} P_{\xi_{1}, \ldots, \xi_{m+1}} A_{\xi_{0}}-P_{\xi_{0}, \ldots, \xi_{m}}<0, \quad \xi_{0}, \ldots, \xi_{m+1} \in\{1, \ldots, M\} .
$$

Clearly, if there exists a solution of the system of LMIs (16) for some $m \in \mathbf{N}$, then solutions exist for every $m^{\prime} \geq m$. In [17, Example 29] Lee and Dullerud present a specific 1-parameter family of UAS switched systems and compute numerically the minimal $m$ required to test the stability of the system. The computations show that $m=0$ is a conservative choice, that is, it does not allow to characterize uniform asymptotic stability. The chosen example "saturates" at $m=7$, that is, the maximal integer $m$ required to check UAS is 7 . It is natural to ask whether examples can be found where the "saturating" $m$ is arbitrarily large (similarly to what happens for the minimal degree of a common polynomial Lyapunov function in the continuous-time case, see [19]). The following proposition gives a positive answer to such question and proposes a construction of UAS systems for which (16) has no solution (for a fixed $m$ ).

Proposition 10 For any fixed integer $m \in \mathbf{N}$ there exists a UAS system of type (1) which does not admit a Lyapunov function of the type (15) satisfying (16).

Proof. The proof works by contradiction. The idea is to consider a continuous-time switched system with suitable properties and to time-discretize it. For every time-step the system that is obtained is stable, but the assumption that all such systems satisfy equations of the form (16) leads to a contradiction when the time-step goes to zero (and the discrete systems converge, roughly speaking, to the continuous one).

Take $d=2$ and $\Xi=\{1,2\}$ and assume by contradiction that if (1) is UAS, then there exist positive definite matrices $P_{\eta_{0}, \ldots, \eta_{m}}$ for every $\left(\eta_{0}, \ldots, \eta_{m}\right) \in\{1,2\}^{m+1}$ such that $(16)$ holds true.

It is well known that there exist uniformly asymptotically stable continuous linear switched systems of the type

$$
\dot{x}=u(t) C_{1} x+(1-u(t)) C_{2} x, \quad u(t) \in\{0,1\}, \quad x \in \mathbf{R}^{2},
$$

such that there exist no positive definite matrix $P$ such that $C_{i}^{T} P+P C_{i} \leq 0$ for $i=1,2$ (see, e.g., [13]). It is possible, moreover, to assume that $C_{1}$ and $C_{2}$ have non-real eigenvalues. (Notice that the exact result appearing in [13] proves the possible nonexistence, for a uniformly asymptotically stable system, of a quadratic Lyapunov function, i.e., of $P>0$ satisfying $C_{i}^{T} P+P C_{i}<0$ for $i=1,2$. Here we impose a slightly stronger property, since we want to rule out the possibility of a positive definite quadratic function which is non-increasing along trajectories of (17). The result, however, directly follows from the reasoning in [13].) 
Let us define, for every $\varepsilon>0$,

$$
A_{1}^{\varepsilon}=e^{\varepsilon C_{1}}, A_{2}^{\varepsilon}=e^{\varepsilon C_{2}},
$$

and consider the corresponding family of discrete systems

$$
x(k+1)=A_{\xi(k)}^{\varepsilon} x(k), \quad \xi(k) \in\{1,2\} .
$$

Every such system is obviously UAS, because of the uniform asymptotic stability of (17). According to the contradiction hypothesis, let us assume that for every $\varepsilon>0$ there exist $P_{\eta_{0}, \ldots, \eta_{m}}^{\varepsilon}>0,\left(\eta_{0}, \ldots, \eta_{m}\right) \in\{1,2\}^{m+1}$, such that (16) holds true. Up to a rescaling we can assume $\max _{\eta_{0}, \ldots, \eta_{m}}\left\|P_{\eta_{0}, \ldots, \eta_{m}}^{\varepsilon}\right\|=1$ for every $\varepsilon>0$. Thus, by compactness, we can find a suitable sequence $\varepsilon_{h} \rightarrow 0$ such that $P_{\eta_{0}, \ldots, \eta_{m}}^{\varepsilon_{\varepsilon}} \rightarrow P_{\eta_{0}, \ldots, \eta_{m}}$ for every $\left(\eta_{0}, \ldots, \eta_{m}\right) \in\{1,2\}^{m+1}$ for some positive semidefinite matrices $P_{\eta_{1}, \ldots, \eta_{m+1}}$. Moreover, there exists at least one $\left(\eta_{0}, \ldots, \eta_{m}\right) \in$ $\{1,2\}^{m+1}$ such that $P_{\eta_{0}, \ldots, \eta_{m}}$ has norm equal to one.

Since, for $\varepsilon>0$ small, we have $A_{i}^{\varepsilon}=\operatorname{Id}+\mathcal{O}(\varepsilon)$ for $i=1,2$, we deduce that

$$
\left(A_{\xi_{0}}^{\varepsilon}\right)^{T} P_{\xi_{1}, \ldots, \xi_{m+1}}^{\varepsilon} A_{\xi_{0}}^{\varepsilon}-P_{\xi_{0}, \ldots, \xi_{m}}^{\varepsilon}=P_{\xi_{1}, \ldots, \xi_{m+1}}^{\varepsilon}-P_{\xi_{0}, \ldots, \xi_{m}}^{\varepsilon}+\mathcal{O}(\varepsilon)<0
$$

for every $\left(\xi_{0}, \ldots, \xi_{m+1}\right) \in\{1,2\}^{m+2}$, so that, passing to the limit along the sequence $\varepsilon_{h}$, we get

$$
P_{\xi_{1}, \ldots, \xi_{m+1}} \leq P_{\xi_{0}, \ldots, \xi_{m}} .
$$

Iterating this inequality $m+1$ times we get

$$
P_{\xi_{m+1}, \ldots, \xi_{2 m+1}} \leq P_{\xi_{0}, \ldots, \xi_{m}} .
$$

Because of the arbitrariness of the $2(m+1)$-tuple $\left(\xi_{0}, \ldots, \xi_{2 m+1}\right)$ in $\{1,2\}^{2 m+2}$, it actually holds

$$
P_{\xi_{m+1}, \ldots, \xi_{2 m+1}}=P_{\xi_{0}, \ldots, \xi_{m}}=: P
$$

for every $\left(\xi_{0}, \ldots, \xi_{2 m+1}\right) \in\{1,2\}^{2 m+2}$, and $\|P\|=1$.

On the other hand, since, for $\varepsilon>0$ small, we have $A_{i}^{\varepsilon}=\operatorname{Id}+\varepsilon C_{i}+\mathcal{O}\left(\varepsilon^{2}\right)$ for $i=1,2$, we deduce that

$$
\left(A_{i}^{\varepsilon}\right)^{T} P_{i, \ldots, i}^{\varepsilon} A_{i}^{\varepsilon}-P_{i, \ldots, i}^{\varepsilon}=\varepsilon\left(C_{i}^{T} P_{i, \ldots, i}^{\varepsilon}+P_{i, \ldots, i}^{\varepsilon} C_{i}\right)+\mathcal{O}\left(\varepsilon^{2}\right)<0,
$$

and dividing by $\varepsilon$ and passing to the limit along the sequence $\varepsilon_{h}$, we get

$$
C_{i}^{T} P+P C_{i} \leq 0
$$

for $i=1,2$. We claim that $P$ is not only semidefinite, but also positive definite. Indeed, assume by contradiction that there exists $v \in \mathbf{R}^{2} \backslash\{0\}$ such that $v^{T} P v=0$. Because of (19), $x^{T} P x$ should be identically equal to 0 along any trajectory of the switched system starting from $v$. Moreover, since $\|P\|=1$, any trajectory starting from $v$ should stay in $\mathbf{R} v$. This would imply that $v$ is an eigenvector of $C_{1}$ and $C_{2}$, which is impossible because $C_{1}$ and $C_{2}$ have non-real eigenvalues.

Thus $P$ is positive definite and satisfies $C_{i}^{T} P+P C_{i} \leq 0$ for $i=1,2$. This contradicts the initial assumption made on $C_{1}, C_{2}$ and the proposition is proved. 


\section{The degenerate case}

We consider here the case in which the non-degeneracy hypothesis appearing in Theorem 6 , namely, the existence of $\bar{\xi} \in \Xi$ such that $A_{\bar{\xi}}$ is invertible, does not hold. The perfect analogue of Theorem 6 is false in this case, as we will see by a counterexample at the end of this section. Different notions of quadratic stability give rise to non-equivalent tests for asymptotic stability. A special role is played by LMIs which hold on the eventual accessible set, which is introduced in the next section.

\subsection{Eventual accessible sets and relaxation of the LMI conditions for stability}

Fix $A_{1}, \ldots, A_{M}$ in $M_{d}(\mathbf{R})$. Define $\Sigma_{0}=\mathbf{R}^{d}$ and

$$
\Sigma_{k}=\cup_{i=1}^{M} A_{i}\left(\Sigma_{k-1}\right), \quad k \in \mathbf{N} .
$$

Then $\Sigma_{k}$ is the set of all points of $\mathbf{R}^{d}$ that can be obtained as evaluation at the time $k$ of a trajectory of the finite system (1).

Lemma 11 Fix $A_{1}, \ldots, A_{M}$ in $M_{d}(\mathbf{R})$ and define $\Sigma_{k}, k \in \mathbf{N}$, as above. Then there exists $\bar{k} \in \mathbf{N}$ such that $\Sigma_{k}=\Sigma_{\bar{k}}$ for every $k \geq \bar{k}$.

Proof. By construction, each $\Sigma_{k}$ is the union of finitely many linear subspaces of $\mathbf{R}^{d}$. We say that a linear subspace $L$ of $\mathbf{R}^{d}$ is a component of $\Sigma_{k}$ if every linear subspace containing $L$ and contained in $\Sigma_{k}$ coincides with $L$.

We associate with each $k \in \mathbf{N}$ and each $\delta \in\{1, \ldots, d\}$, the number $\nu(\delta, k)$ of components of $\Sigma_{k}$ of dimension $\delta$. For every $k \in \mathbf{N}$, consider the set $D_{k}=\{\delta \mid \delta \in\{1, \ldots, d\}, \nu(\delta, k) \geq 1\}$, and rewrite its terms in decreasing order $D_{k}=\left\{d_{1}^{k}>d_{2}^{k}>\cdots>d_{l_{k}}^{k}\right\} \subset\{1, \ldots, d\}$.

A simple inductive argument shows that $\Sigma_{k+1}$ is contained in $\Sigma_{k}$. In particular $d_{1}^{k}$ is nonincreasing as a function of $k$ and there exists therefore $\bar{k}$ such that $d_{1}^{k}$ is constant for $k \geq \bar{k}$. Moreover, $\nu\left(d_{1}^{k}, k\right)$ is non-increasing for $k \geq \bar{k}$. Henceforth, up to eventually taking a larger $\bar{k}, \nu\left(d_{1}^{k}, k\right)$ is also constant for $k \geq \bar{k}$. That means that the union of the components of $\Sigma_{k}$ of maximal dimension is a constant set for $k \geq \bar{k}$. In particular, its image through $A_{i}$ is constant for every $i$, implying that $d_{2}^{k}$ is non-increasing for $k \geq \bar{k}$. The same argument as above shows that, up to increasing $\bar{k}$, the union of the components of $\Sigma_{k}$ of dimension $d_{2}^{k}$ is a constant set for $k \geq \bar{k}$. Iterating the argument finitely many times, we get that $\Sigma_{k}$ is constant for $k$ large enough.

Lemma 11 allows to associate with a finite family of matrices $\left\{A_{1}, \ldots, A_{M}\right\}$ in $M_{d}(\mathbf{R})$ the eventual accessible set $\Sigma_{\infty}\left(A_{1}, \ldots, A_{M}\right)=\Sigma_{\bar{k}}$, where $\bar{k}$ is as in the statement of the lemma. By construction, $\Sigma_{\infty}\left(A_{1}, \ldots, A_{M}\right)$ is invariant for $A_{1}, \ldots, A_{M}$ and, moreover,

$$
\Sigma_{\infty}\left(A_{1}, \ldots, A_{M}\right)=\cup_{i=1}^{M} A_{i}\left(\Sigma_{\infty}\left(A_{1}, \ldots, A_{M}\right)\right) .
$$

Notice that this notion of accessible set is different from others appearing in the literature (see for instance [21]), where the initial point is usually fixed and the switching law is not the only controlled parameter. 
Remark 12 Following the procedure of the proof of Lemma 11, one can explicitly find an upper bound on $\bar{k}$ which depends on $d$ and $M$ only. Hence, $\Sigma_{\infty}\left(A_{1}, \ldots, A_{M}\right)$ can be computed algorithmically in finitely many steps.

From now on, given a finite system of type (1), we write $\Sigma_{\infty}\left(A_{1}, \ldots, A_{M}\right)=\cup_{h=1}^{s} V_{h}$ where $s \in \mathbf{N}, V_{h}=T_{h}\left(\mathbf{R}^{d_{h}}\right)$ and $T_{h}: \mathbf{R}^{d_{h}} \rightarrow \mathbf{R}^{d}$ is a linear injective map for $h=1, \ldots, s$. Since, by definition, every trajectory of (1) lies inside $\Sigma_{\infty}\left(A_{1}, \ldots, A_{M}\right)$ after a finite number of steps, the existence of a Lyapunov function defined only on $\cup_{h=1}^{s} V_{h}$ guarantees the asymptotic stability of the system. This observation leads to the following result which introduces a relaxed version of the LMIs corresponding to (5)-(6) in the degenerate case.

Proposition 13 Let (1) be finite. If there exist $M$ symmetric matrices $P_{1}, \ldots, P_{M}$ such that

$$
\begin{aligned}
& T_{h}^{T} P_{i} T_{h}>0, \quad i=1, \ldots, M, \quad h=1, \ldots, s, \\
& T_{h}^{T}\left(A_{i}^{T} P_{j} A_{i}-P_{i}\right) T_{h}<0, \quad i, j=1, \ldots, M, \quad h=1, \ldots, s,
\end{aligned}
$$

then system (1) is uniformly asymptotically stable.

\subsection{Comparison between different notions of quadratic stability in the degenerate case}

In the degenerate case PTD-quadratic stability can be expressed by means of LMIs (see Theorem 14 below) that are in general not equivalent to those obtained in Section 3.1 for nondegenerate systems. These new LMIs are the same as those introduced in Proposition 13, except for condition (20), which is replaced by the stronger requirement that each $P_{i}$ is positive definite.

Theorem 14 Let (1) be finite. Then (1) is PTD-quadratically stable if and only if there exist $P_{1}, \ldots, P_{M}$ positive definite such that (21) holds true.

Proof. Assume first that there exist $P_{1}, \ldots, P_{M}>0$ statisfying (21). Define $v(x, \xi)=x^{T} P_{\xi} x$ for $x \in \mathbf{R}^{d}$ and $\xi=1, \ldots, M$. Let $\bar{k}$ be as in the statement of Lemma 11 . In order to show that (1) is PTD-quadratically stable it is then enough to take the Lyapunov function $(k, x, \xi) \mapsto V(k, x, \xi)$ of the form $V(k, x, \xi)=\varphi(k) v(x, \xi)$ with $\varphi(k)=\beta^{\max (\bar{k}-k, 0)}$ and $\beta$ large enough.

Now assume that (1) is PTD-quadratically stable and fix $(k, \xi, x) \mapsto V(k, \xi, x)$ and $(k, \xi) \mapsto$ $P_{k, \xi}$ as in Definition 5. Denote by $\Sigma_{\infty}$ the set $\Sigma_{\infty}\left(A_{1}, \ldots, A_{M}\right)$. The proof can then be concluded following exactly the same steps as in the proof of Theorem 6: equation (10) can be proved to hold on $\Sigma_{\infty}$ and the same compactness argument which is used to prove (11) implies that there exist $\hat{\alpha}>0$ and $P_{1}^{*}, \ldots, P_{M}^{*}>0$ such that $A_{i}^{T} P_{j}^{*} A_{i}-P_{i}^{*} \leq-\hat{\alpha}$ Id on $\Sigma_{\infty}$ for every $i, j \in\{1, \ldots, M\}$, proving (21).

When $\Sigma_{\infty}$ is linear, $P D$-quadratic stability on $\Sigma_{\infty}$ (in the sense of Proposition 13) is equivalent to PD-quadratic stability on the entire $\mathbf{R}^{d}$, as proved below. The same is not true in general, as illustrated by a counterexample at the end of this section.

Proposition 15 Let (1) be finite. Assume that $\Sigma_{\infty}=\Sigma_{\infty}\left(A_{1}, \ldots, A_{M}\right)$ is a linear subspace of $\mathbf{R}^{d}$. Assume that there exist $M$ symmetric matrices $P_{1}, \ldots, P_{M}$ satisfying (20) and (21). Then (1) is PD-quadratically stable. 
Proof. Define recursively $\Sigma_{\infty}^{k}$, for $k \in \mathbf{N}$, in such a way that $\Sigma_{\infty}^{0}=\Sigma_{\infty}$ and

$$
\Sigma_{\infty}^{k+1}=\cap_{i=1}^{M} A_{i}^{-1}\left(\Sigma_{\infty}^{k}\right), \quad k \in \mathbf{N} .
$$

Notice that $A_{i}\left(\Sigma_{\infty}\right) \subset \Sigma_{\infty}$ for every $i=1, \ldots, M$. Hence, $\Sigma_{\infty}^{0} \subset \Sigma_{\infty}^{1}$. By recurrence we get $\Sigma_{\infty}^{k} \subset \Sigma_{\infty}^{k+1}$ for every $k \in \mathbf{N}$.

Moreover, the non-decreasing sequence of linear spaces $\Sigma_{\infty}^{k}$ reaches $\mathbf{R}^{d}$ in a finite number of steps. Indeed, let $k$ be such that $\cup_{j \in \mathbf{N}} \Sigma_{\infty}^{j}=\Sigma_{\infty}^{k}$ and assume by contradiction that $\Sigma_{\infty}^{k} \neq \mathbf{R}^{d}$. Hence, for every $x \in \mathbf{R}^{d} \backslash \Sigma_{\infty}^{k}$ one has $x \notin \cap_{i=1}^{M} A_{i}^{-1}\left(\Sigma_{\infty}^{k}\right)$, so that there exists $i \in\{1, \ldots, M\}$ such that $A_{i} x \notin \Sigma_{\infty}^{k}$. This means that, starting from any point $x$ outside $\Sigma_{\infty}^{k}$ there exists a sequence $\left\{i_{j}\right\}_{j \in \mathbf{N}} \subset\{1, \ldots, M\}$ such that $A_{i_{j}} A_{i_{j-1}} \cdots A_{i_{1}} x \notin \Sigma_{\infty}^{k}$ for every $j \in \mathbf{N}$. This contradicts the characterization of $\Sigma_{\infty}$ given in Lemma 11, which would imply that there exist $\bar{k} \in \mathbf{N}$ such that

$$
A_{i_{\bar{k}}} A_{i_{\bar{k}-1}} \cdots A_{k_{1}} x \in \Sigma_{\infty} \subset \Sigma_{\infty}^{k}
$$

We claim that for every $k$ there exist $P_{1}^{(k)}, \ldots, P_{M}^{(k)}>0$ such that $A_{i}^{T} P_{j}^{(k)} A_{i}-P_{i}^{(k)}<0$ on $\Sigma_{\infty}^{k}$ for every $i, j \in\{1, \ldots, M\}$. The thesis of the proposition follows taking $k=\bar{k}$.

For $k=0$ the assertion is true because of the hypothesis of the proposition. Indeed one can set $P_{i}^{(0)}=P_{i}+\hat{P}_{i}$ where the positive semidefinite symmetric matrix $\hat{P}_{i}$ satisfies $\hat{P}_{i}=0$ on $\Sigma_{\infty}$ and $\hat{P}_{i}>\left\|P_{i}\right\|$ Id on the orthogonal space to $\Sigma_{\infty}$, denoted by $\Sigma_{\infty}^{\perp}$. Assume that the assertion is true for a given $k \geq 0$. We look for matrices $P_{1}^{(k+1)}, \ldots, P_{M}^{(k+1)}>0$ in the form $P_{j}^{(k+1)}=P_{j}^{(k)}+\hat{P}_{j}^{(k+1)}$, where $\hat{P}_{j}^{(k+1)}$ is positive semidefinite and $\hat{P}_{j}^{(k+1)}=0$ on $\Sigma_{\infty}^{k}$. Let $\varepsilon_{k}, R_{k}>0$ be such that $A_{i}^{T} P_{j}^{(k)} A_{i}-P_{i}^{(k)} \leq-\varepsilon_{k}$ Id on $\Sigma_{\infty}^{k}$ and $\left\|A_{i}^{T} P_{j}^{(k)} A_{i}-P_{i}^{(k)}\right\| \leq R_{k}$ for every $i, j \in\{1, \ldots, M\}$. We have

$$
\begin{aligned}
x^{T}\left(A_{i}^{T} P_{j}^{(k+1)} A_{i}-P_{i}^{(k+1)}\right) x= & x_{1}^{T}\left(A_{i}^{T} P_{j}^{(k)} A_{i}-P_{i}^{(k)}\right) x_{1}+x_{2}^{T}\left(A_{i}^{T} P_{j}^{(k)} A_{i}-P_{i}^{(k)}\right) x_{2} \\
& +2 x_{1}^{T}\left(A_{i}^{T} P_{j}^{(k)} A_{i}-P_{i}^{(k)}\right) x_{2}-x_{2}^{T} \hat{P}_{i}^{(k+1)} x_{2} \\
\leq & -\varepsilon_{k}\left\|x_{1}\right\|^{2}+R_{k}\left(\left\|x_{2}\right\|^{2}+2\left\|x_{1}\right\|\left\|x_{2}\right\|\right)-x_{2}^{T} \hat{P}_{i}^{(k+1)} x_{2},
\end{aligned}
$$

where, for $x \in \Sigma_{\infty}^{k+1}$, we considered the decomposition $x=x_{1}+x_{2}$, with $x_{1} \in \Sigma_{\infty}^{k}$ and $x_{2} \in \Sigma_{\infty}^{k+1} \cap\left(\Sigma_{\infty}^{k}\right)^{\perp}$. Thus, by choosing $\hat{P}_{i}^{(k+1)}$ in such a way that $\hat{P}_{i}^{(k+1)}>\left(R_{k}+R_{k}^{2} / \varepsilon_{k}\right) \operatorname{Id}$ on $\Sigma_{\infty}^{k+1} \cap\left(\Sigma_{\infty}^{k}\right)^{\perp}$ we get that $A_{i}^{T} P_{j}^{(k+1)} A_{i}-P_{i}^{(k+1)}<0$ on $\Sigma_{\infty}^{(k+1)}$, completing the proof of the proposition.

Proposition 15 has the following corollary, in the spirit of Theorem 6 .

Corollary 16 Let (1) be finite and assume that $\Sigma_{\infty}=\Sigma_{\infty}\left(A_{1}, \ldots, A_{M}\right)$ is a linear subspace of $\mathbf{R}^{d}$. Then (1) is PTD-quadratically stable if and only if it is PD-quadratically stable if and only if there exist $M$ symmetric matrices $P_{1}, \ldots, P_{M}$ satisfying (20) and (21).

Proof. Let (1) be PTD-quadratically stable. Notice that the restriction of (1) to $\Sigma_{\infty}$ is a welldefined, nondegenerate, PTD-quadratically stable system. Theorem 6 then implies that it is PD-quadratically stable. By extending the quadratic forms yielding PD-quadratic stability by zero on $\Sigma_{\infty}^{\perp}$, we get $M$ symmetric matrices $P_{1}, \ldots, P_{M}$ satisfying (20) and (21). Proposition 15 implies that system (1) is PD-quadratically stable.

The converse implication being trivial, the corollary is proved. 
Example 17 We conclude the section by noticing that Proposition 15 cannot be extended in general to the case where $\Sigma_{\infty}$ is not linear. A counterexample can be constructed as follows. Take $d=3$ and

$$
A_{\Xi}=\left\{A_{1}, A_{2}\right\}, \quad A_{1}=\left(\begin{array}{ccc}
\lambda & 0 & 0 \\
0 & 0 & 0 \\
0 & 0 & 0
\end{array}\right), A_{2}=\left(\begin{array}{ccc}
-1 & 0.3 & -3 \\
0.5 & 0 & 1.5 \\
0 & -0.3 & 0
\end{array}\right) .
$$

Clearly, for $\lambda \neq 0, \Sigma_{\infty}$ is the union of the plane $\operatorname{span}\left\{-2 e_{1}+e_{2}, e_{1}-e_{3}\right\}$ and the line $\mathbf{R} e_{1}$.

It can be checked numerically (for instance by using the package yalmip for matlab) that if $\lambda \geq 0.863$ then the system is not PD-quadratically stable. On the other hand, by taking the positive definite matrices

$$
P_{1}=\left(\begin{array}{ccc}
10.6 & 5.4 & 1.3 \\
5.4 & 18.3 & -0.2 \\
1.3 & -0.2 & 20.2
\end{array}\right), \quad P_{2}=\left(\begin{array}{ccc}
12.4 & 9.6 & 17.9 \\
9.6 & 38.3 & -22.8 \\
17.9 & -22.8 & 89.6
\end{array}\right)
$$

one can check that, if $|\lambda| \leq 0.868$, then (21) is satisfied with $s=2$ and

$$
T_{1}=e_{1}, \quad T_{2}=\left(\begin{array}{cc}
-2 & 1 \\
1 & 0 \\
0 & -1
\end{array}\right) .
$$

In particular if $\lambda \in[0.863,0.868]$ then the system is PTD-quadratically stable but not PDquadratically stable.

Consider now the polytopic system corresponding to $A_{\Xi}=\operatorname{conv}\left\{A_{1}, A_{2}\right\}$, where $A_{1}, A_{2}$ are defined as above. For every $\lambda \neq 0$ this system is nondegenerate, in the sense of Remark $\%$. Hence PTD-quadratic stability and PD-quadratic stability (and poly-quadratic stability) are equivalent. In particular, they fail to hold for $\lambda \in[0.863,0.868]$. However, the uniform asymptotic stability of the system can be deduced from the first part of Proposition 4.

In general, then, the uniform asymptotic stability of a polytopic system can be tested by the LMIs (20) and (21) which are less conservative that the usual inequalities (5) and (6).

\section{Conclusion}

We compared different notions of stability for discrete-time switched systems of type (1). We first proved (Section 3.1) that, if these exists $\bar{\xi} \in \Xi$ such that $A_{\bar{\xi}}$ is invertible, looking for a quadratic Lyapunov function in its more general form $V(k, \xi, x)=x^{T} P(k, \xi) x$ (that is, in a class which depends on an infinite number of parameters) is equivalent to looking for it in the much smaller class $V(\xi, x)=x^{T}\left(\sum_{i=1}^{M} \xi_{i} P_{i}\right) x$ (which depends on finitely many parameters). Such equivalence does not hold in general when the modes corresponding to the vertices of $\Xi$ are degenerate. In the latter case, we proposed a relaxation of the LMI test for stability based on the notion of eventual accessible set. We also discussed the problem of detecting through quadratic Lyapunov functions the stability of polytopic switched systems whose switching functions have some common bound on the speed of variation. 


\section{References}

[1] A. A. Ahmadi, R. M. Jungers, P. Parrilo, and M. Roozbehani. Analysis of the joint spectral radius via Lyapunov functions on path-complete graphs. In Proceedings of HSCC'11, 2011.

[2] F. Amato, M. Mattei, and A. Pironti. Gain scheduled control for discrete-time systems depending on bounded rate parameters. Internat. J. Robust Nonlinear Control, 15(11):473-494, 2005.

[3] J.-P. Aubin and A. Cellina. Differential inclusions, volume 264 of Grundlehren der Mathematischen Wissenschaften [Fundamental Principles of Mathematical Sciences]. SpringerVerlag, Berlin, 1984. Set-valued maps and viability theory.

[4] R. Barmish. Stabilization of uncertain systems via linear control. IEEE Trans. Automat. Contr., 28(8):848-850, 1983.

[5] P. H. Bauer, K. Premaratne, and J. Durán. A necessary and sufficient condition for robust asymptotic stability of time-variant discrete systems. IEEE Trans. Automat. Control, 38(9):1427-1430, 1993.

[6] F. Blanchini and S. Miani. Stabilization of LPV systems: State feedback, state estimation, and duality. SIAM journal on control and optimization, 40(1):76-97, 2004.

[7] V. D. Blondel and Y. Nesterov. Computationally efficient approximations of the joint spectral radius. SIAM Journal of Matrix Analysis, 27(1):256-272, 2005.

[8] S. Boyd, L. E. Ghaoui, E. Feron, and V. Balakrishnan. Linear Matrix Inequalities in System and Control Theory. SIAM, Studies in Applied Mathematics, 1994.

[9] A. Casavola, D. Famularo, and G. Franzè. A feedback min-max MPC algorithm for LPV systems subject to bounded rates of change of parameters. IEEE Trans. Automat. Control, 47(7):1147-1153, 2002.

[10] F. Colonius and W. Kliemann. The dynamics of control. Systems \& Control: Foundations \& Applications. Birkhäuser Boston Inc., Boston, MA, 2000. With an appendix by Lars Grüne.

[11] J. Daafouz and J. Bernussou. Parameter dependent Lyapunov functions for discrete time systems with time varying. Systems and Control Letters, 43(8):355-359, 2001.

[12] J. Daafouz, P. Riedinger, and C. Iung. Stability analysis and control synthesis for switched systems: a switched Lyapunov function approach. IEEE Trans. Automat. Control, 47(11):1883-1887, 2002.

[13] W. P. Dayawansa and C. F. Martin. A converse Lyapunov theorem for a class of dynamical systems which undergo switching. IEEE Trans. Automat. Control, 44:751-760, 1999.

[14] J. C. Geromel and P. Colaneri. Robust stability of time varying polytopic systems. Systems and Control Letters, 55(1):81-85, 2006. 
[15] M. Jungers, R. C. L. F. Oliveira, and P. L. D. Peres. MPC and LPV systems with bounded parameter variations. Internat. J. Control, 84(1):24-36, 2011.

[16] R. Jungers. The joint spectral radius, volume 385 of Lecture Notes in Control and Information Sciences. Springer-Verlag, Berlin, 2009. Theory and applications.

[17] J.-W. Lee and G. E. Dullerud. Uniform stabilization of discrete-time switched and Markovian jump linear systems. Automatica J. IFAC, 42(2):205-218, 2006.

[18] H. Lin and P. J. Antsaklis. Stability and stabilizability of switched linear systems: a survey of recent results. IEEE Trans. Automat. Control, 54(2):308-322, 2009.

[19] P. Mason, U. Boscain, and Y. Chitour. Common polynomial Lyapunov functions for linear switched systems. SIAM journal on control and optimization, 45:226-245, 2006.

[20] A. P. Molchanov and Y. S. Pyatnitskiy. Criteria of asymptotic stability of differential and difference inclusions encountered in control theory. Systems Control Lett., 13(1):59-64, 1989.

[21] P. Santesso and M. E. Valcher. Monomial reachability and zero controllability of discretetime positive switched systems. Systems Control Lett., 57(4):340-347, 2008.

[22] R. Shorten, F. Wirth, O. Mason, K. Wulff, and C. King. Stability criteria for switched and hybrid systems. SIAM Review, 49:545-592, 2005.

[23] J. Theys. Joint spectral radius: Theory and approximations, 2005. PhD Thesis.

[24] J. L. Willems. Stability Theory of Dynamical Systems. NELSON, 1970. 